Historic, Archive Document

Do not assume content reflects current scientific knowledge, policies, or practices. 



\section{Tree, Shrub and Fruit Seeds Ornamentaliand Forestry, Purposes ${ }^{8 t o n}, D . C$. C 20}

\section{PRICE LIST \\ 1912-1913}

CONYERS B. FLEU, JR.

TREE SEEDSMAN

GERMANTOWN, PHILADELPHIA

U. S. A. 


\title{
Tree and Shrub Seeds
}

The general method to persue in the propogation of trees and shrubs from seed is to plant those seeds of Conifers and Evergreen Trees in the Spring, while those of a Deciduous nature in the Fall. Carefully select a fine, sandy loam and make the beds three feet in width and sow the seeds broadcast; after sowing cover to the depth of about the thickness of the seed with light sandy soil and gently press the beds with the back of a spade to bring the earth in contact with the seeds. Shade is an essential, as seeds germinate best in darkness; this can be accomplished by either making lath sash or covering them with forest leaves, keeping them in place by a light skeleton frame. The leaves or sash should be removed as soon as the seedlings begin to appear, as light is needed.

To preserve seed of Conifers and Evergreen Trees until the proper sowing season, mix them in dry sand. Should it be impossible to plant the Deciduous seeds in the Fall, they will keep in excellent condition by mixing them in slightly moist soil; keep the soil in a damp condition until ready to sow.

Deciduous seeds which have hard shells such as Gymnocladus, honey and black locust, if not sown in the Fall, are best soaked for a day or so and then at once sown.

NOTICE! Every effort is extended to secure and collect only the very best seeds, but it is known that some seeds are occasionally wormy, such as Quercus, and Castanea. This defect, however, cannot be detected until after the worm has eaten its way out, which often is after the seeds have been shipped. While on the other hand, there are trees such as Liriodendron, Sugar Maple, and etc., that never perfect all their seeds. The imperfect seeds can not be separated. With this in view and the many known and unknown causes which prevent seeds from germinating, all seeds are sold without any warranty, expressed or implied, as to quality, productiveness or any other matter of any seeds, and in no way will I be responsible for crop conditions. All orders are accepted on these conditions. Should any cause for complaint arise on receipt of shipment notice must be sent within one week of same, otherwise it is understood that the goods are accepted.

CONDITIONS. As I collect but few more seeds than are ordered, all offers and acceptances of orders are subject to the condition of the season's crop, and failing to get the seeds expected or the quantities ordered from my growers and collectors, I reserve the right to reduce proportionately all orders that have been booked.

SHIPPING FACILITIES. Small orders can be sent to all parts of the world by sample post and to nearly every country by parcel post. Shipments are dispatched very quickly owing to my warehouse being closely connected with the forwarders of New York and Philadelphia ports. The cost of postage, bags and boxes is charged additionally.

TERMS. My terms are cash with order or satisfactory references must be supplied.

RATES. One quarter bushel sold at the bushel price, one quarter pound sold at the pound rate.

SPECIAL PRICES. Those requiring large quantities of seeds will be given special prices.

\author{
CONYERS B. FLEU, Jr. \\ TREE SEEDSMAN \\ GERMANTOWN, PHILADELPHIA \\ U. S. A. \\ A, B, C CODE \\ 4th \& 5th Edition
}

CABLE ADDRESS

FLEU, PHILA. 
Abies (Picea) amabilis, California Fir ...........\$4 00 Arizonica, Arizona Cork Fir............... $1000 \quad 100$ balsamea, Ba!sam Fir .............. 150 brachyphylla ................. 350

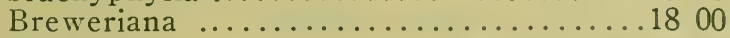

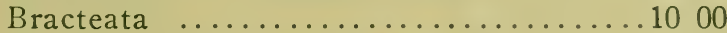
concolor, California Silver Fir........ 225 concolor, Colorado Silver Fir......... 200 firma, Japanese .................. 250 Fraseri, Double Balsam.............. 350 grandis, Pacific Coast Fir ........... 325 magnifica, Pacıfic Coast Fir............ 350 Mariesii, Japan Silver Fir ........... 450 nobilis, Pacific Coast Fir............. 350 Nordmanniana, Nordman Fir........... 150 pectinata, European Silver Fir............ 125 sachalinensis, Saghalien Fir .......... 500 sub-alpina ...................... 450 Umbellata ...................... 400 Veitchii ......................... 400

Araucaria excelsa ........................ 200

Arctostaphylos glauca ..................... 150

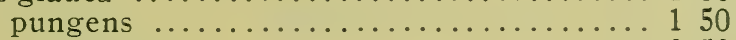
tomentosa ........................ 250 uva ursi ........................ 300

Berberis Aquifolium (Mahonia) ............... 125

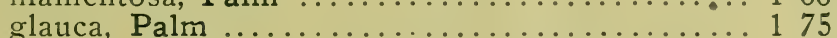

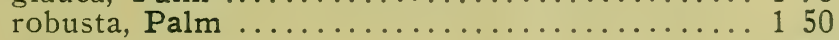

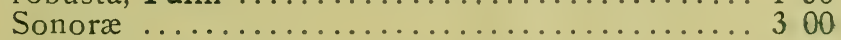

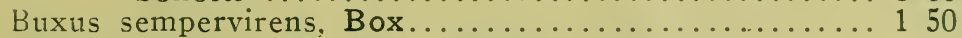

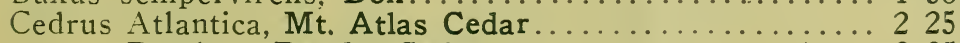

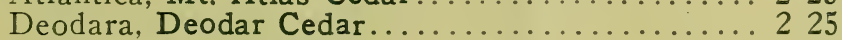

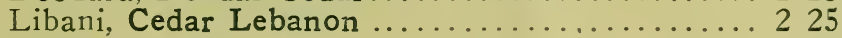

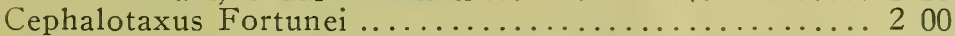

Cinnamomum Camphora, Camphor Tree.............. 125

Crataegus Pyracantha, Evergreen Thorn................ 100

Cryptomeria Japonica, Japanese Cedar................ 150

Cupressus Arizonica ............................. 450

Goveniana ............................... 300

Guadalupensis .....................4 400

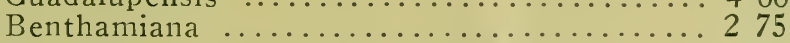

Knightiana ................................... 300

Lawsoniana, Lawson's Cypress............. 300

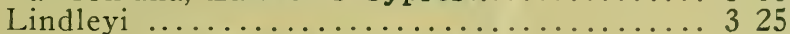

macrocarpa, Monterey Cypress .................. 125

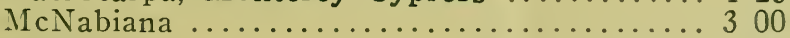

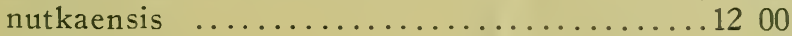

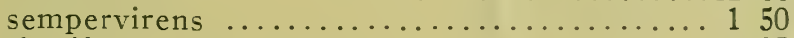

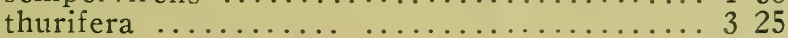

thyoides, White Cedar .................... 375

Eucalyptus amygdalina ............ per packet, $\$ 025 \quad 800$

corynocalyx ................ $25 \quad 800$

globulus ............ "

Gunnii ............... " " $25 \quad 500$

leucoxylon ............. " $\quad 25 \quad 700$

obtusifolia ........... " " $25 \quad 1000$

resinifera $\ldots \ldots \ldots \ldots \ldots \ldots$ " $\quad 25 \quad 800$

robusta .............. " " $25 \quad 400$

rostrata $\ldots \ldots \ldots \ldots \ldots \ldots$ " $\ldots \ldots \ldots$

viminalis ............ " $\quad 25 \quad 450$ 
Hex Aquifolium, English Holly............... Per Iu. P'er uz.

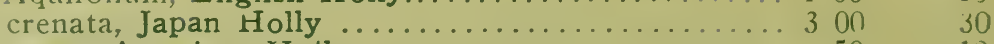

opaca, American Holly.................... $50 \quad 10$

Juniperus Chinensis, Chinese Juniper................ $150 \quad 15$

Californica .............................. $300 \quad 30$

communis, Common Juniper.............. $100 \quad 10$

monosperma ...................... $125 \quad 15$

occidentalis, Western .................2 $00 \quad 20$

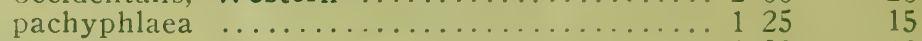

prostrata ........................ $100 \quad 10$

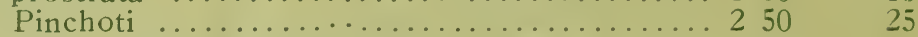

rigida, Japan Cedar .................. $350 \quad 35$

sabinoides ......................... $225 \quad 25$

scopulorum ........................ 150

Sabina, Savin Juniper ................. 150

suecica, Swedish Juniper ............... $150 \quad 15$

Virginiana, Red Cedar ............... $75 \quad 10$

Kalmia angustifolia, Laurel ..................... $500 \quad 50$

latifolia, Sheep Laurel................... $500 \quad 50$

Laurus nobilis, Sweet Bay ................... $100 \quad 15$

Libocedrus decurrens ........................ $300 \quad 30$

. Iagnolia grandiflora, Evergreen Magnolia............ 1 $25 \quad 15$

Nuttallia cerasiformis ...................... $300 \quad 30$

Oreodaphne Californica, Cal. Nutmeg............... $100 \quad 10$

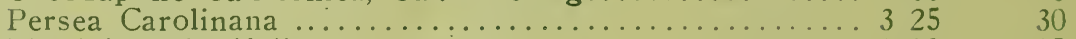

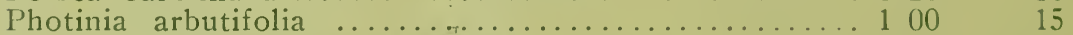

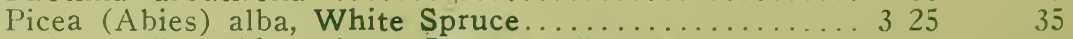

Alcoquiana, Japanese Spruce ....... $450 \quad+5$

ajanensis .................... $450 \quad 45$

bicolor, from Japan .............. $450 \quad 50$

Engelmanni .................. 425

excelsa, Norway Spruce............. 125

Glehnii, from Japan............... $600 \quad 60$

Hookeriana .................... $800 \quad 75$

nigra, Black Spruce.............. 400 40

Orientalis, Oriental Spruce .......... $350 \quad 35$

pungens, Colorado Blue Spruce........ $400 \quad$ t()

polita .......................4 400

rubra, Red Spruce................. 350

Sitchensis (Menziesii) .............. $350 \quad 35$

l'inus aristata, Colorado Foxtail Pine............... $400 \quad 35$

Austriaca, Austrian Pine .................... $150 \quad 15$

Banksiana, Jack Pine ........................ 5 on 50

Canariensis, Canary Island Pine ............ $250 \quad 25$

cembra. Swiss Pine ........................ $100 \quad$ 1()

cembroides, Mexican Stone Pine ............ 425

contorta ......................... 550 . 50

Coulteri, Big Cone Pine .................. $300 \quad 30$

lensiflora, Japanese.Pine ................ 200

edulis, Pinyon Pine...................... 150

excelsa, Himalayan Pine.................... $250 \quad 35$

flexilis, Colorado Pine .............................. 300

insignis, Monterey Pine.................. $250 \quad 25$

Jeffreyi ................................ 300

Koraiensis ............................ $250 \quad 25$

Lambertiana, Sugar Pine .............

Laricio ............................... 2525

Massoniana ............................. 200

mitis .......................... $400 \quad 40$

monophylla, Single Leaf Pine............ $250 \quad 25$

monticola, Pacific Coast White Pine ........... 450

Mughus, Dwarf Mountain Pine ............. $250 \quad 25$

muricata ......................... 450

Murryana, Lodgepole Pine ............... $600 \quad 50$

Nelsoni ............................4 $50 \quad 50$

palustris, Yellow Broom Pine ............... 300

Parryana, Mexican Pinyon .................. 4 nn th 
Pinus Pentaphylla, from Japan.................. 400

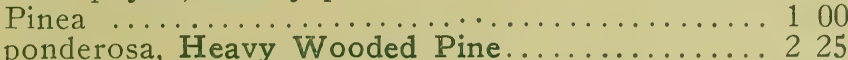

ponderosa (from Colorado) (scopulorum)........... 250

pumila, from Japan ..................... 400

resinosa, Red, Norway Pine .............. 750

rigida, Pitch Pine...................... 275

Sabiniana, Pine ..................... 150

Strobus, White Pine .................. 200

sylvestris, Scotch Pine .................... 200

taeda, Loblolly Pine ................... 425

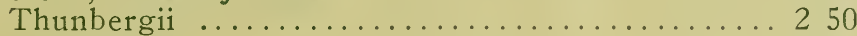

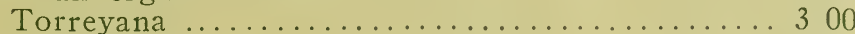

tuberculata ..........................4 50

Podocarpus Chinensis ..................... 250

Prinos glaber, Ink Berry .................. 100

Prunus (Cerasus) Caroliniana ................... 75

" " ilicifolia, Holly Leaf Cherry........ 100 integrifolia, Entire Leaf Cherry..... 150

Pseudotsuga Douglasii, Douglas Spruce ................. 3500

$\begin{array}{ll}3 & 50 \\ 4 & 50\end{array}$

macrocarpa .................... 450

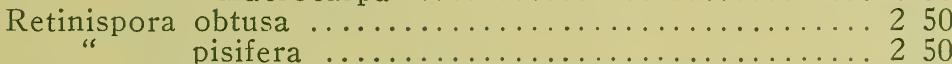

Rhododendron Catawbiense, Mountain Laurel........ 400 maximum " " ....... 300

Sabal Adansoni, Palm ........................ 100

" Palmetto, Palm ........................ 100

" serrulata, Saw Palm .................. 100

Sciadopitys verticillata, Umbrella Pine ........... 350

Sequoia gigantea, California Big Tree........... 700

Taxus baccata, English Yew .................... 150

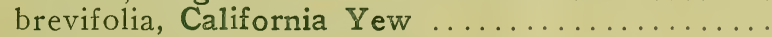

cuspidata ....................... 350

Thuja gigantea, Western Arbor-vitae....... 350 occidentalis, American orientalis, Chinese

Thujopsis Dolabrata (From Japan) .............. 600

Torreya Californica ....................... 150 nucifera (From Japan) ..................... 150

Tsuga (Abies) Canadensis, Hemlock Spruce......... 400 Diversifolia (From Japan) ............... 450 heterophylla ......................... 700 Mertensiana, Western $\ldots \ldots \ldots \ldots$ " $\ldots \ldots \ldots \ldots \ldots$. 00 Pattoniana ............................. 700

Sieboldi, Japanese

Ulex Europaea, Furze ....................... 150

Yucca angustifolia, Adam's Needle ............... 225
" baccata
brevifolia
filamentosa
Whipplei

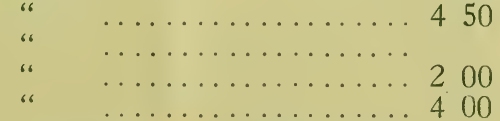

\title{
DECIDUOUS TREES AND SHRUBS.
}

\author{
Acer campestre. \\ circinatum \\ dasycarpum, Silver \\ Ginnale \\ glabrum \\ macrophyllum, Oregon \\ platanoides, Norway \\ pseudo-platanus, Sycamore \\ rubrum, Red \\ saccharinum, Sugar \\ spicatum, Mountain
}

English Maple......... Per 75

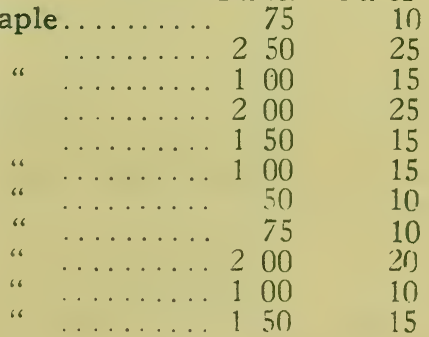


Acer striatum, Striped

Per lb.
150

Ailanthus glandulosa. Tree of Heaven.............. 50

Albizzia Julibrissin, Mimosa or Paradise Tree........ 150

Alnus glutinosa, Alder ...................... 75

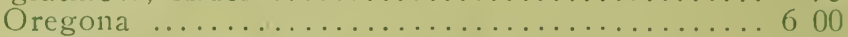

serrulata, Swamp Alder ............... 75

Amelanchier Botryapium ................... 150

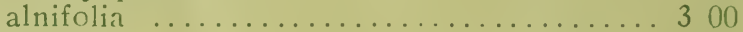

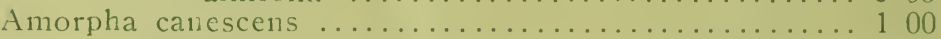

fruiticosa, Indigo Shrub ............... 100

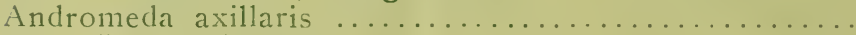

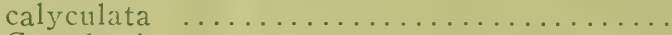

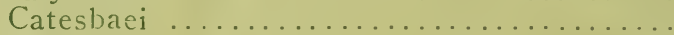

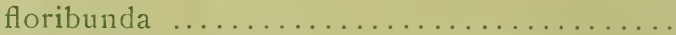

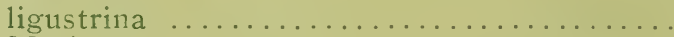

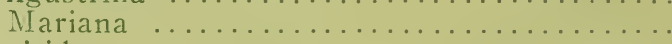

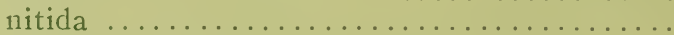

racemosa $\ldots \ldots \ldots \ldots \ldots \ldots \ldots \ldots \ldots \ldots \ldots$

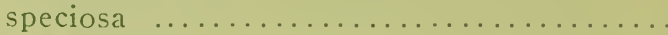

Aralia Sieboldi (From Japan)................. 250

spinosa, Angelica Tree ....................... 200

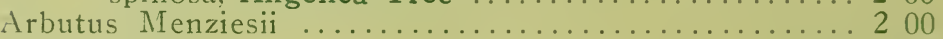

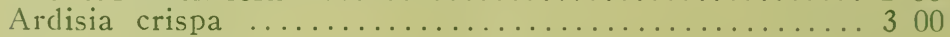

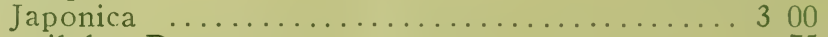

Asimina triloba, Papaw ...................... 75

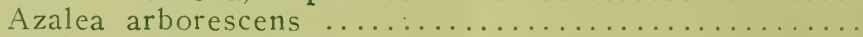

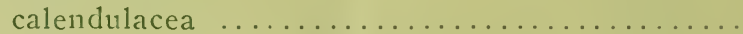

mollis

nudiflora

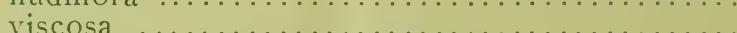

Baccharis halimifolia, Groundsel Shrub

Berberis Fremonti, Silver-leaved Barberry ........... 500

nervosa .......................... 425

Thunbergii, Japanese Barberry ............. 125

vulgaris, Common Barberry ............. 100

purpurea, Purple Barberry......... 150

Betula alba, European White Birch................. 100

excelsa, Yellow " $\ldots \ldots \ldots \ldots \ldots \ldots \ldots \ldots .200$

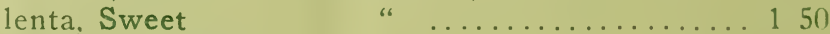

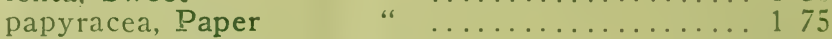

populifolia, Poplar $\quad$ “ $\ldots \ldots \ldots \ldots \ldots \ldots \ldots \ldots . .100$

rubra, $\operatorname{Red} \quad$ " .................. 150

Callicarpa Americana ..................... 85

purpurea ................... 200

Calycanthus floridus laevigatus. Sweet Shrub ........... 100 occidentalis, Western "

Camellia Japonica .......................... 1 5()

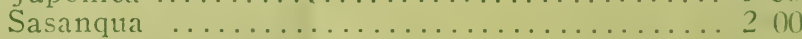

Caragana arborescens, Siberian Pea .............. 125

Carpenteria Californica

Carpinus Americana.

Hornbeam............. 75

Betulus, European $\quad$. $\quad \ldots \ldots \ldots \ldots \ldots \ldots$ (0)

Carya alba, Shellbark Hickory . per bil-hel. $\$ 5$ on 15

amara, Bitter-nut

olivæerormis, Pecan-nut

4 (0) 15

porcina, Pig-nut

+() $0-15$

sulcata, West'n Shellbark " $\quad$ " $\quad$ " $\quad+0015$

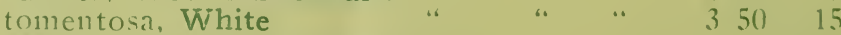

Cassia Marilandica. Wild Senna ................ 100

Catalpa bignonioides, Eastern Catalpa ............. 1 50

speciosa. Western Catalpa. This is the true forest trec. speciosa, guaranteed true to name. 225

Cearothus Americanus (clean) ................. 225

integerrinus $\ldots \ldots \ldots \ldots \ldots \ldots \ldots \ldots \ldots \ldots+50$

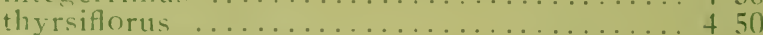

reltis nccidentalis. Nettle Tree

$+50 \quad 50$


Cephalanthus occidentalis $\ldots \ldots \ldots \ldots \ldots \ldots \ldots \ldots \ldots$

Cerasus. See Prunus.

Cercis Canadensis, Judas Tree ................. 100

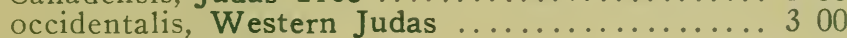

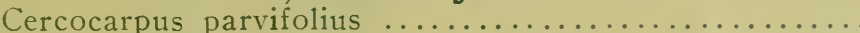

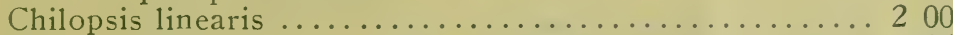

Chionanthus Virginica, White Fringe ........... 125

Citrus trifoliata, Hardy Orange ................. 200

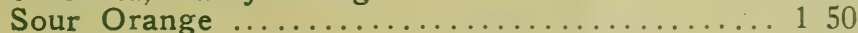

Cladrastis tinctoria, Virgilia ............... 350

Clethra alnifolia, Sweet Pepper Bush............. 100

Colutea arborescens ......................... 100

Cornus alternifolia, Blue-berried Dogwood .......... 75

florida, Large White Dogwood ........... 50

Mas, Cornelian Cherry ................... 75

Nuttalli, Pacific Coast Dogwood............. 150

paniculata ...................... 150

sericea, Silky Dogwood ................ 100

Stolonifera .......................... 100

Corylus Americana, Hazel Nut ............... 75

Cratægus coccinea, White Thorn, berries........... 75 cordata, Washington " crus-galli. Cockspur " " ............. 75

oxycantha, English Thorn, berries.......... 75

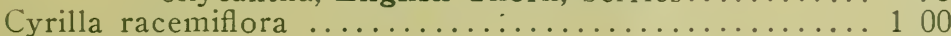

Cytisus Scoparius, Scotch Broom .................. 100

Diospyros Kaki, Japan Persimmon ................ 150

Virginiana, Persimmon ................. 75

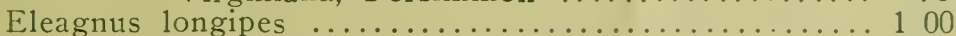

Euonymus Americanus, Burning Bush ........... 125

atropurpureus, " " ............ 125

Exochorda grandiflora, Pearl Bush .............. 250

Fagus ferruginea, American Beech ............... 100

sylvatica, European " .................. 125 purpurea, Blood-leaved ............ 275

Fraxinus Americana,

White Ash............. 100

excelsior, European " ........... 75

Oregona $\quad \ldots \ldots \ldots \ldots \ldots 200$

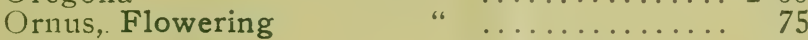

pubescens, Red

quadrangulata, Blue

sambucifolia, Black

$\ldots \ldots \ldots \ldots \ldots \ldots \ldots$

viridis, Green “ $\ldots \ldots \ldots \ldots \ldots \ldots .75$

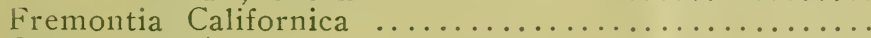

Garrya elliptica ...................... 750

Gaylussacia resinosa, Huckleberry ................. 125

Gleditschia triacanthos, Honey Locust .............. 50

Gordonia lascanthus ...................... 150

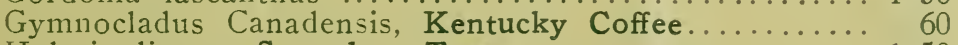

Halesia diptera, Snowdrop Tree ................. 150 tetraptera "

Hamamelis Virginica, Witch Hazel ................... 125

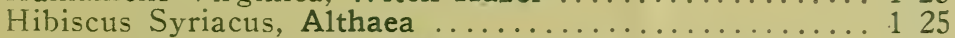

Hippophæa rhamnoides, Sea Buckthorn............. 150

Hydrangea arborescens ...................... 150

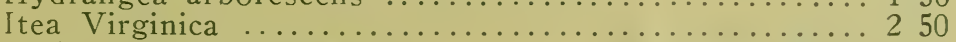

Juglans cinerea, Butternut ............ per bushel, $\$ 25015$

Californica ............... 50

cordiformis, Japan Walnut ... • 50

nigra, Black Walnut........ " " $250 \quad 15$

regia, English " ........ " " 50030

rupestris, Arizona Walnut .... “ “ " $1000 \quad 50$

Sieboldii (From Japan) ................. 50

Koelreuteria paniculata, Varnish Tree ............... 150

Larix Americana, American Larch. Price on application.

Europæa, European Larch ................. 225

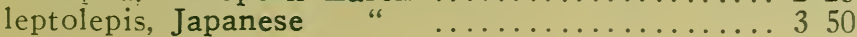


Larix occidentalis, Western Larch ...................

Laburnum vulgare ............................. 125

Lindera Benzoin, Spice Bush ................... 75

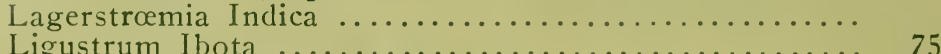

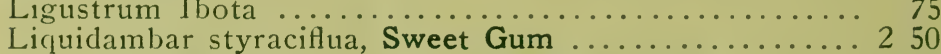

Liriodendron tulipifera, Tulip Tree ................. 100

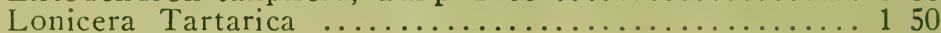

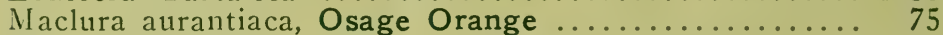

Magnolia acuminata ...................... 150

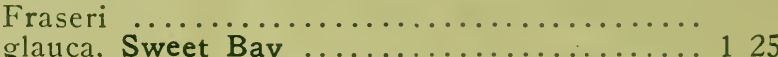

grandiflora, Evergreen ................. 125

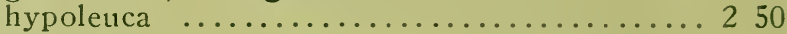

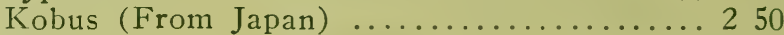

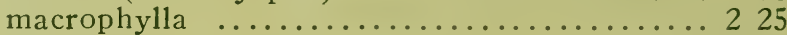

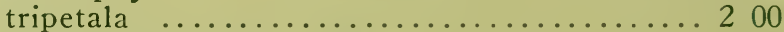

Melia umbraculiformis, China Tree................. 100

Morus alba, White Mulberry .................. 150 rubra

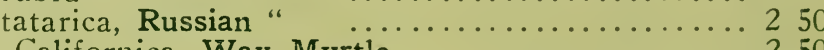

Myrica Californica, Wax Myrtle .............. 250

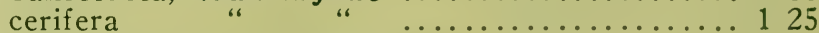

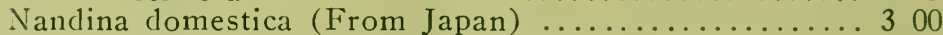

Negundo aceroides, Ash-leaved Maple (Western)........ 100

Nyssa aquatica, Sour Gum.................... 125 multiflora " " ................ 75

uniflora, Tupelo, Sour Gum .............. 100

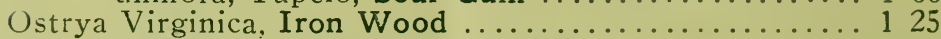

Oxydendrum arboreum (Andromeda) ............. 300

Paulownia imperialis, Empress Tree ............ 150

Phellodendron Amurense .................... 75

Platanus occidentalis, American Plane .............. 75

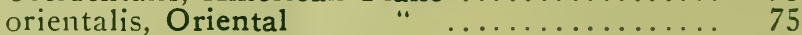

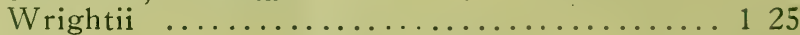

Populus Wislizeni ......................... 125

Prinos verticillatus, Deciduous Holly .................... 100

Prosopis juliflora, pods, Mesquite Bean ............. 125 pubescens, pods, Tornio Bean ............. 125

Prunus Americana, Wild Plum .................. 75

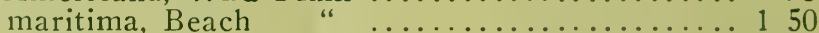
Pennsylvanica ....................... 100 serotina, Wild Cherry .................... 50

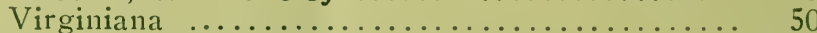

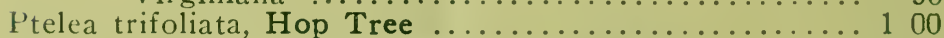

Pyrus Americana, American Mountain Ash........... 150

Aucuparia, European " “ ............. 100

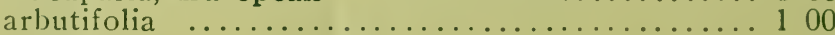

coronaria ... ....................... 50

rivularis, Oregon Crab ....................... 300

agrifolia

acpuatica

bicolor, Meadow

Cateshai

Cerris, Turkey

chrysolepis

cinerea, Upland Willow Oak

coccinea. Scarlet

falcata, Spanish

Oak.

Garryana

ilicifolia, Scrub, imbricaria, Laurel laurifolia, Southern Laurel Oak lyrata 
DECIDUOUS TRESS AND SHRUBS-Continued.

Quercus nigra, Black Jack obtusiloba, Post

palustris, Pin

Phellos, Willow

Prinus, Chestnut

Robur, English

rubra, Red

Texana

tinctoria Black

Vislizeni
$750 \quad 35$

$6 \quad 50 \quad 40$

$600 \quad 25$

$400 \quad 20$

$700 \quad 50$

$450 \quad 25$

100

200

125

100

100

300

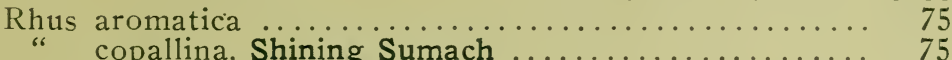

cathartica

Frangula

Purschiana

175

75

200

75

700

400

400

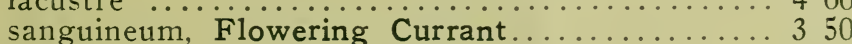

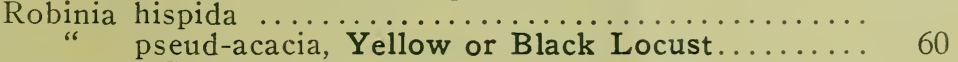

Romneya Coulteri

100

200

100

400

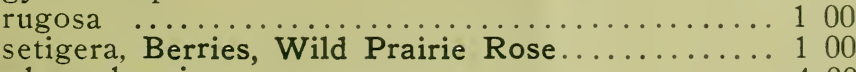

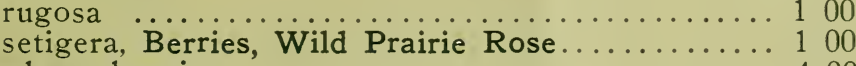

Rubus leucodermis .......................... 400

nvtkanus .........................6 00

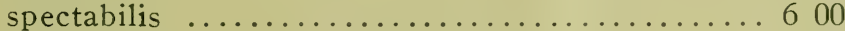

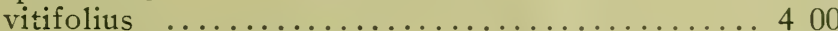

Salisburia adiantifolia, Ginkgo ................... 100

Sambucus Canadensis, Elder ................ 75 glauca .......................... 250

racemosus

Sapindus marginatus, Soap-berry ............... 150

Sassafras officinale ...................... 100

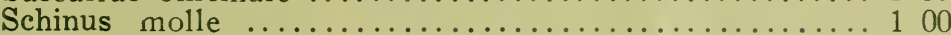

Sophora Japonica, Pagoda Tree.................... 125

Spiræa opulifolia ...................... 300

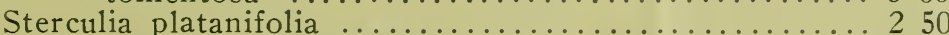

Staphylea trifolia, Bladder-nut ................... 125

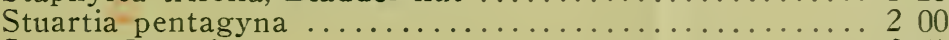

Styrax Japonica .......................... I 50

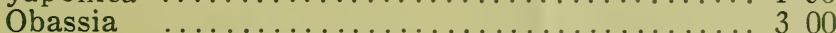

Syringa villosa, Chinese Lilac ....................... 150 vulgaris, Common Lilac ......................... 125

Taxodium distichum, Deciduous Cypress ............. 100

Tilia Americana, American Linden .............. 75

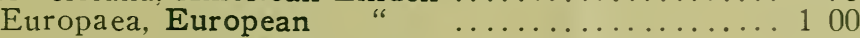

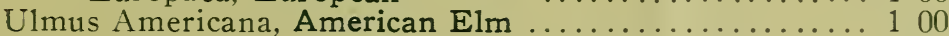
campestre. European " ....................... 125

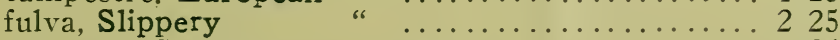
montana, Scotch "

Vaccinium arboreum, Bush Huckleberry .......... 150 corymbosum " " ........... 300 macrocarpon, Cranberry, clean seed ............

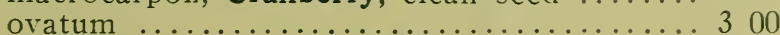
Pennsylvanicum, Huckleberry ............ 150 
DECIDUOUS TREES AND SHRUBS-Continued.

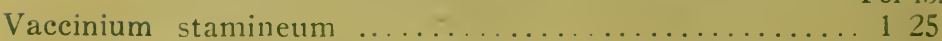

Viburnum acerifolium ............................ 100

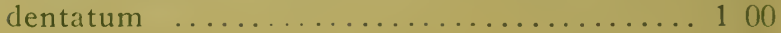

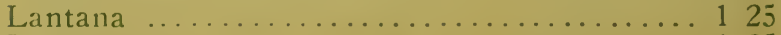

Lentago ....................... 125

nudum ....................... 125

Oxycoccos .................... 100

prunifolium ..................... 100

Zanthoxylon fraxinæfolium, Prickly Ash ............ 150

\section{VINES AND CLIMBERS.}

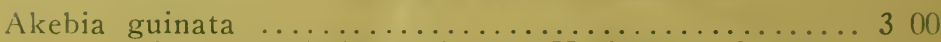

Ampelopsis heterophylla variegata, Variegated Grape Veitchii, Japan Creeper ..............\$1 00 Virginica, Virginia “ $\ldots \ldots \ldots \ldots \ldots \ldots \ldots 100$

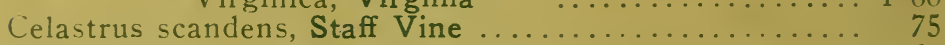

Clematis flammula, Sweet Clematis ............ 150

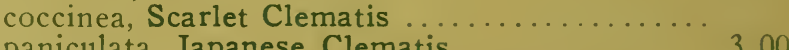

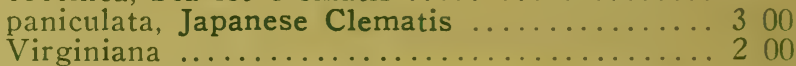
vitalba, English Clematis ............ 125

Hedera Helix. Ivy ........................ 100

Menispermum Canadense, Moon Seed ............ 150

Passiflora incarnata, Passion Flower ............... 275

Pueraria Thumbergiana, Kudzu Vine ............ 450

Smilax glauca ......................... 175

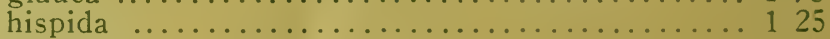

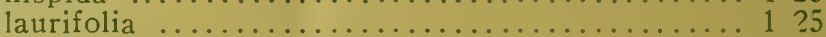

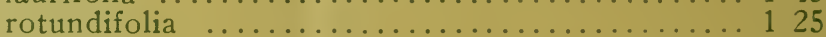

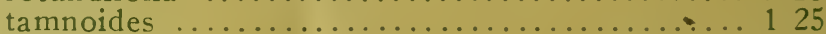

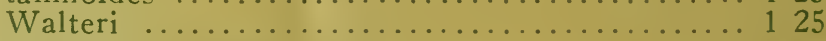

Tecoma radicans, Trumpet Vine .............. 150

Vitis. See Fruits.

Wistaria frutescens .......................... 150

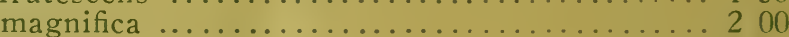

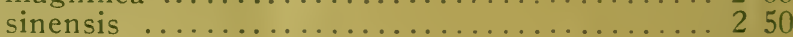

\section{FRUITS.}

Apple, French Crab. Prices per bushel on application. 50

Cherry Mahaleb, $\$ 18.00$ per bu.; .3 bus. at $\$ 17.00$ per bu... 50

Chestnut, American ............................ 30

" f Spanish..................................... 35

Grape, Vitîs æstivalis .................... 100 coignetiæ Japánese Wild Grape ........4 400 cordifolia ...................... 100 labrusca ..................... 100 riparia .................... 100

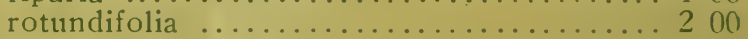

Hazel Nut, American ...................... 75

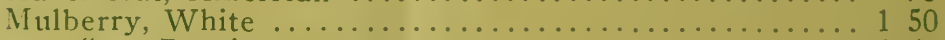

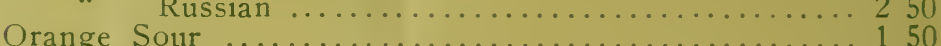

Peach pits. Natural, per bu., $\$ 2.75 \ldots \ldots \ldots \ldots \ldots \ldots \ldots .15$

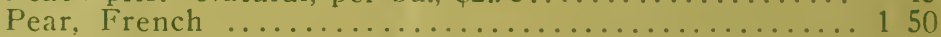

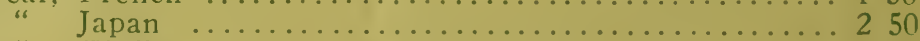

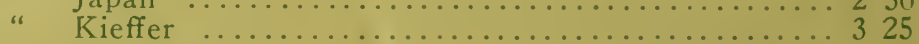

Pecan Nut ............................ ber bushel, $\$ 80030$

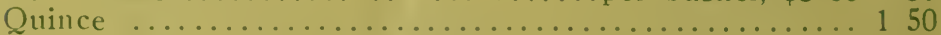

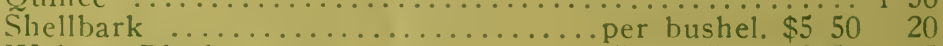

Walnut, Black .................. " 250 " 5

Butternut ................ " 25015

California ....................... 50

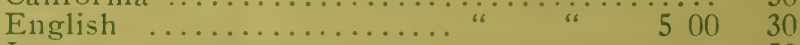

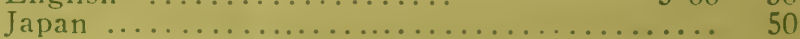

Where seeds are to go by mail, add $9 \mathrm{cts}$. per lb. to cover postage. 